\title{
IMPORTANT VECTOR-BORNE DISEASES WITH THEIR ZOONOTIC POTENTIAL: PRESENT SITUATION AND FUTURE PERSPECTIVE
}

\author{
M. A. H. N. A. Khan \\ Department of Pathology, Faculty of Veterinary Science \\ Bangladesh Agricultural University, Mymensingh-2202, Bangladesh
}

\begin{abstract}
SUMMARY
Vector-borne diseases (VBDs) of zoonotic importance are the global threat in the human life and on animal welfare as well. Many vector-borne pathogens (VBPs) have appeared in new regions in the past two decades, while many endemic diseases have increased in incidence. Although introductions and emergence of endemic pathogens are often considered to be distinct processes, many endemic pathogens are actually spreading at a local scale coincident with habitat change. Key differences between dynamics and diseases burden result from increased pathogen transmission following habitat change, deforestation and introduction life into new regions. Local emergence of VBPs are commonly driven by changing in ecology (deforestation, massive natural calamities, civil wares etc.), altered human behavior, enhanced enzootic cycles, pathogen invasion from anthropogenic trade and travel, genomic changes of pathogens to coup up with the new hosts, vectors, and climatic conditions and adaptability in wildlife reservoirs. Once a pathogen is established, ecological factors related to vector and host characteristics can shape the evolutionary selective pressure and result in increased use of people as transmission hosts. West Nile virus (WNV), Nipah virus and Chikungunya virus (CHIKV) are among the best-understood zoonotic vector-borne pathogens (VBPs) to have emerged in the last two decades and showed just how explosive epidemics can be in new regions. Zoonotic VBPs that are likely introduced into new regions include Rift Valley Fever and Japanese Encephalitis viruses (JEV) in the Americas, Venezuelan equine encephalitis virus in Eurasia or Africa, Crimean-Congo Hemorrhagic Fever virus (CCHFV) in new parts of Eurasia. Vector-borne diseases currently impose global burden on public health and animal welfare including widespread formerly zoonotic human diseases, such as malaria, leishmania and dengue fever, as well as zoonotic diseases for which humans are dead end hosts, such as Lyme disease, WNV and CCHF. It requires highly equipped laboratory facilities and technical manpower to address emergence and re-emergence of vector-borne zoonotic diseases. Financial and technological hurdles persist in developing countries, making diagnosis and control facility difficult where these diseases are stubbornly most prevalent. Development of technological and highly knowledgeable manpower is the key to protect public health and eco-health. An awareness building about the changing risk of VBPs to prevent introduction foreign pathogens is far more difficult because this is commonly an inevitable consequence of the globalization of trade and travel and in most cases is accidental. Designing of active surveillance of the deadly infectious pathogens by combining the expertise of veterinary and human health could play pivotal roles towards reducing burden of VBPs. History suggests that successful control of VBPs requires prompt identification, swift action, mobilization of fund for developing technical expertise and occasionally by using draconian social measures.
\end{abstract}

Keywords: Vector-borne diseases, zoonotic potential, future perspective

\section{Vectors and vector-borne diseases (VBDs):}

The important vectors transmitting zoonotic diseases are mosquitos, flies, fleas, lice, biting flies, bugs, ticks, mites, snails, helminthes etc. which actively transmit pathogens (Table 1) from an infected reservoir host animal to another individual. Many of these are blood sucker (Figs. 1 \& 2), which ingest disease-producing microorganisms during a blood meal from an infected host (human or animal) and later inject it into a new host during their subsequent blood meal. Mosquitoes are the best known vector for transmission of a number of diseases (Samad, 2011). Vector-borne diseases are transmitted in two ways; mechanical and biological. In mechanical transmission, the disease causing agents are transported by flying insects (without any development in the vector) through contaminated legs, wings, mouthparts or through excretory materials (e.g. Anthrax, typhoid, Q fever). In the biological transmission the disease causing agents complete a development cycle in the vectors before transmission in to the vertibrate hosts. Vector-borne diseases are the major illnesses of man and animals now a day and caused by pathogens and parasites. Every year more than 1 billion vector-borne diseases develop globally with a case fatality of 1 million lives (Gubler, 2009).

Corresponding e-mail address: hadi.khan@bau.edu.bd 
Zoonosis: The diseases and infections, which are naturally transmissible between vertebrate animals and human are zoonotic and this phenomenon is refered to as zoonosis. Major impacts of zoonoses include illness, monetary loss, adverse effect on morale of personnel, unfavorable publicity and medico-legal implications. The common routes of transmission of zoonotic diseases are bites of insects and through faeces, urine, saliva, blood, semicooked food, milk, aerosole, oral and contact of infected individuals. There are thousands of diseases transmissible between animals and human and bears tremendous social reparcation and impose restriction on national and international trade. Zoonoses may be bacterial, viral, or parasitic, or may involve un-conventional agents. The important classes of zoonotic diseases are meta-zoonosis, transmitted biologically by the invertebrate's vectors. The impact of zoonoses mostly depends upon the nature of aetiologic agent (very virulent, virulent, moderately virulent), ranges of reservoir hosts and life cycles in the vectors and hosts as well.

\section{Risk factors associated with vector-borne zoonosis:}

1. Globalization of trade and travel, unplanned urbanization and environmental devastation due to climate change poses significant impact on VBDs transmission in recent years

2. Due to lack of awareness of some VBDs like dengue, chikungunya, Nipah and West Nile virus etc. have emerged in countries where they were previously unknown

3. Changes in agricultural practices, changes in the breeding places of vectors due to deforestation, draughts, variation in temperature and rainfall affect increase transmission or wipe out few of the VBDs

4. Lack of proper education, improve awareness and monitoring about the distribution of vectors, vector-borne diseases and other climate-sensitive diseases

5. A crucial element in catching vector-borne diseases is the behavioural change of curious people during travel and tourism and searching for feed and living places in wild environment

6. Poor national economy and lack of mobilizing fund for developing technical expertise

7. Lack of draconian measures often emerge or reemerge VBDs in developing worlds

Globally the important VBPs with zoonotic potentials included leishmania, malaria, dengue, nipah virus, elephantiasis, schistosomiasis, trypanosomiasis, chagas disease, yellow fever, Japanese encephalitis, onchocerciasis etc. Few of the diseases were noted briefly for better undestanding the host range, vectors involved (Figs. 1 \& 2), epidemiology, ecology and distribution pattern, that may enable the epidemiologist to desig future preventive and control stretagies (Samad, 2011).

Leishmaniasis: Leishmaniasis (dom dom fever) are protozoan parasites of great medical and veterinary significance and transmitted to a susceptible host by infected female phlebotomine sand flies (Torres, 2006, 2007). The disease is endemic in Bangladesh since the begening of 19th century and now a day is a big challange for public health. Out of 30 reported species of leishmania, over 20 can infect humans and is transmitted to humans by $~ 30$ different species of phlebotomine sandflies (Bailey and Lockwood, 2007). Leishmaniasis in human consists of four main clinical syndromes; a. cutaneous leishmaniasis (most common) b. muco-cutaneous leishmaniasis (also known as espundia) c. visceral leishmaniasis (VL, popularly known as Kala-azar, most serious form) and d. post-kala-azar dermal leishmaniasis (PKDL, chronic condition). VL is caused by two leishmanial species, L. donovani or L. infantum, depending on the geographical area. Leishmania infantum infects mostly children and immunosuppressed individuals, whereas L. donovani infects all age groups.

Natural vertebrate hosts of Leishmania parasites are humans, armadillos, sloths, dogs, cats, hyraxes, rats, gerbils, monkeys, opossums, horses, foxes, golden jackals etc. (Khan et al., 2012). Visceral leishmaniasis is prevalent throughout the tropical and sub-tropical regions of Africa, Asia (Samad, 2011), the Mediterranean, southern Europe (old world) and South and Central America (new world, Gonzalez et al., 2009). The disease in human is endemic in 61 countries and is responsible for the annual disability of 1.3 million people and 20000 to 30000 deaths annually. Greater than $90 \%$ of VL cases occur in six countries including Bangladesh, Ethiopia, Brazil, India, Nepal and Sudan (http://www.who.int/mediacentre/factsheets/fs375/en/2015), while the equivalent number of cases of cutaneous leishmaniasis occur in Afghanistan, Algeria, Brazil, Iran, Peru, Saudi Arabia and Sudan (Khanjani et al., 2009). 
Table 1. The important vectors and vector-borne zoonotic diseases distributed worldwide. Leishmaniasis, malaria, elephantiasis, dengue, heartworm, rabies and nipah are prevailing in Bangladesh. Recent trend is to import high yielding dairy cattle from Australia. Gochihata Aquaculture Farms limited, Kishorgonj import hundreds of dairy cattle from Australia (1991-1992). Recently Milk vita, Sirajgonj and RDA, Bogra started importing dairy cattle from Australia without much test/ test certificate on it, and Q fever could have present over there.

\begin{tabular}{|c|c|c|c|}
\hline Type of vectors & Diseases transmitted & Pathogens & Principal hosts \\
\hline Sand flies & Leishmaniasis & $\begin{array}{l}\text { L. donovani } \\
\text { L. braziliensis }\end{array}$ & Man, jackals and dogs \\
\hline Anopheles & Malaria & $\begin{array}{l}P . \text { falciparum } \\
P . \text { vivax, } P . \text { malariae }\end{array}$ & Man and primates \\
\hline Aedes & Dengue & Dengue virus & Man and primates \\
\hline $\begin{array}{l}\text { Culex, Anopheles } \\
\text { Mansonia and Aedes }\end{array}$ & $\begin{array}{l}\text { Lymphoid Filariasis/ } \\
\text { Elephantiasis }\end{array}$ & Wuchereria bancrofti & $\begin{array}{l}\text { Human is the only known } \\
\text { natural host }\end{array}$ \\
\hline Musca & Eye worm & Loa loa & Man, cattle and dogs \\
\hline Aedes & Heart worm & Dirofilaria immitis & Man, canids and felids \\
\hline $\begin{array}{l}\text { Snails } \\
\text { (Biomphalaria } \\
\text { and Bulinus) }\end{array}$ & Schistosomiasis & $\begin{array}{l}\text { S. mansoni, } \\
\text { S. haematobium } \\
\text { S. japonicum }\end{array}$ & $\begin{array}{l}\text { Cattle, water buffalo, } \\
\text { horses, dogs, cats, rodents } \\
\text { and monkeys }\end{array}$ \\
\hline $\begin{array}{l}\text { Tsetse fly } \\
\text { (Glossina genus) }\end{array}$ & $\begin{array}{l}\text { Trypanosomiasis } \\
\text { (sleeping sickness) }\end{array}$ & $\begin{array}{l}\text { Trypanosoma brucei } \\
\text { rhodesiense }\end{array}$ & $\begin{array}{l}\text { Human, wild ruminants and } \\
\text { domestic cattle }\end{array}$ \\
\hline Fruit bats & Nipah & Nipah virus & Human \\
\hline $\begin{array}{l}\text { Bats, secretion of } \\
\text { infected hosts }\end{array}$ & Ebola & Ebola virus & Human and primates \\
\hline $\begin{array}{l}\text { Bite of infected } \\
\text { mammals, bats }\end{array}$ & Rabies & $\begin{array}{l}\text { Rabies virus } \\
\text { (Lyssa viruses) }\end{array}$ & All mammals \\
\hline Culex & Japanese encephalitis & $\begin{array}{l}\text { Japanese encephalitis } \\
\text { virus }\end{array}$ & Human, pigs, ducks \\
\hline Aedes & Chikungunya & Chikungunya virus & Human and primates \\
\hline Aedes & Yellow fever & Yellow fever virus & Human, primates \\
\hline Ticks and mosquitoes & West Nile virus & West Nile virus & Human, birds, reptiles \\
\hline $\begin{array}{l}\text { Culex and } \\
\text { Culiseta }\end{array}$ & $\begin{array}{l}\text { Western equine } \\
\text { encephalomyelitis }\end{array}$ & Alphavirus & $\begin{array}{l}\text { Mammals, birds, reptiles \& } \\
\text { amphibians }\end{array}$ \\
\hline Mosquitoes & $\begin{array}{l}\text { St. Louis } \\
\text { encephalitis }\end{array}$ & Flaviviridae & $\begin{array}{l}\text { Human, birds, monkey, } \\
\text { white mice }\end{array}$ \\
\hline Ticks (Hyalomma sp) & $\begin{array}{l}\text { Crimean-Congo } \\
\text { haemorrhagic fever }\end{array}$ & $\begin{array}{l}\text { Bunyaviridae } \\
\text { (RNA viruses) }\end{array}$ & $\begin{array}{l}\text { Human, cattle, sheep, goats, } \\
\text { ostriches }\end{array}$ \\
\hline $\begin{array}{l}\text { Rat flea } \\
\qquad \text { (Xenopsylla cheopsis) }\end{array}$ & Plague & Yersinia pestes & $\begin{array}{l}\text { Human, rats, guineapigs, } \\
\text { other rodents }\end{array}$ \\
\hline Ticks (Ixodes species) & Lyme disease & Borrelia burgdorferi & Human, rodent, deer \\
\hline Ticks, Flies & Q fever & Coxiella burnetii & Cattle, sheep, goat, human \\
\hline Ticks (Dermacentor) & $\begin{array}{l}\text { Rocky Mountain } \\
\text { spotted } \\
\text { fever }\end{array}$ & Rickettsia rickettsii & Human, rodents \\
\hline $\begin{array}{l}\text { Ticks } \\
\text { (Ixodes scapularis) }\end{array}$ & Babesiosis & $\begin{array}{l}\text { Babesia microti } \\
\text { B. divergens } \\
\text { B. duncani }\end{array}$ & $\begin{array}{l}\text { Human, mice, small } \\
\text { mammals }\end{array}$ \\
\hline
\end{tabular}




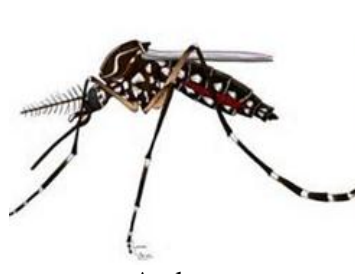

Aedes

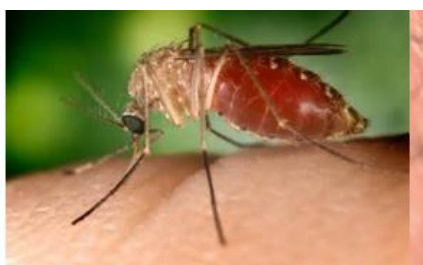

Anopheles

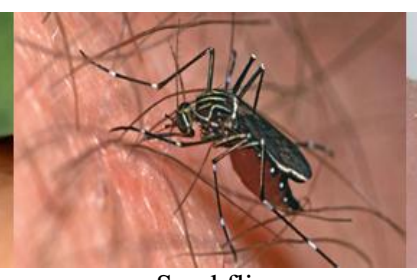

Sand flies

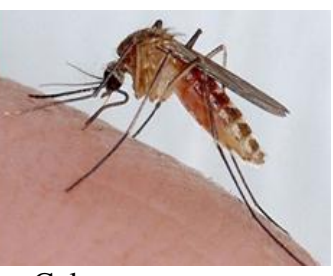

Culex

Fig. 1. Various vector mosquitoes played role in transmitting a number of VBDs (Sources https://www.google.com.bd/\#q=mosquitoes).

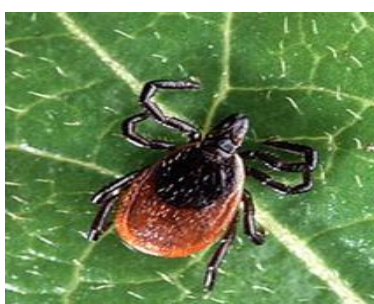

Deer tick

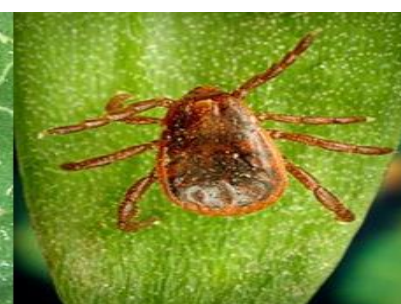

Brown tick

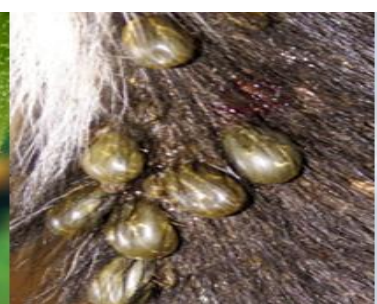

Cattle tick

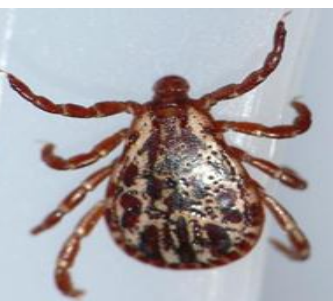

American dog tick

Fig. 2. Various vector ticks https://www.google.com.bd/\#q=ticks).
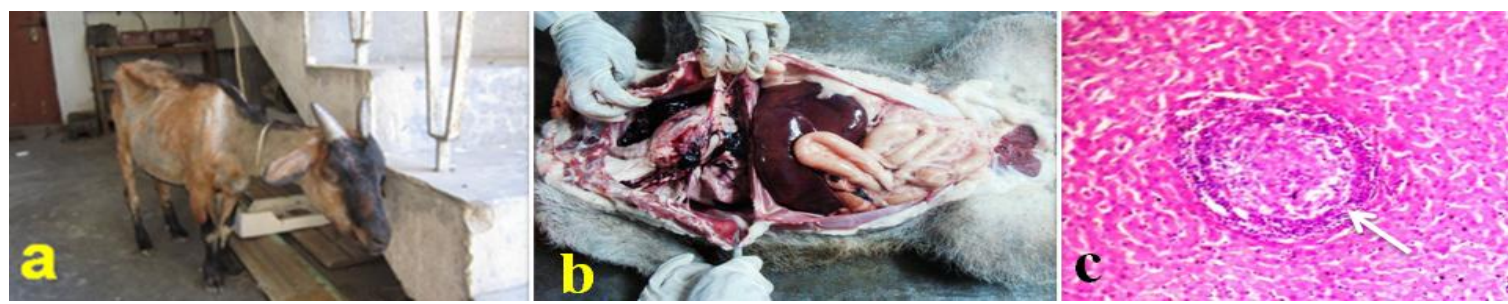

Fig. 3. Goat (a) and Jackal (b) carrying Leishmania donovani a causal organism of VL in their internal organs in Bangladesh. Typical granulomatous nodule lacking caseous necrotic center was seen in the liver (c, white arrow) of apparently healthy jackal (H\&E, 10x).

The disease affects the poorest people in the community, and is associated with malnutrition, population displacement, poor housing, weak immune systems and lack of resources (Mondal et al., 2008). Its spread is linked to migration and environmental changes such as deforestation, building of dams, irrigation schemes and urbanization. Depending on the types of leishmaniasis, the disease can cause fever, weight loss, enlargement of the spleen and liver, anaemia, rashes and skin ulcers. Leishmaniasis is treatable and curable. Early diagnosis and treatment reduces the spread of the disease and can prevent disabilities and death. In visceral leishmaniasis, diagnosis is made by combining clinical signs with parasitological or serological tests (rapid diagnostic tests and others). In cutaneous and mucocutaneous leishmaniasis serological tests have limited value. In cutaneous leishmaniasis, clinical manifestation with parasitological tests and nucleic acid based tests confirms the diagnosis. Prevention and control of leishmaniasis require a combination of strategies including control of sandflies and their animal reservoir (including dogs, jackals, foxes, small ruminants and cattle), improving livelihood conditions and personal protection against sand fly bites. The sandflies are about $3 \mathrm{~mm}$ in length, sucks blood at night and use of mosquitoes net can not prevent man and animals from sandflies bite. It requires cleaning all the jungle, bushes, dark corner of the house, tunnels and brick stakes and spraying insecticide very often in that places to reduce densities of sandflies and prevent occurance of leishmaniasis. 
The emergence of zoonotic visceral leishmaniasis (ZVL) as an increasing public health problem is mainly due to demographic and ecological factors. ZVL now occurs in many regions of Latin America where it was not previously reported. In South America, especially in Brazil, Colombia and Venezuela, migration and urbanization have largely contributed to the increasing public health problem posed by ZVL. A prime example is the massive migration that was occurred in north-eastern Brazil (year 1990-92) due to a long-lasting drought and its socio-economic consequences (ruined crops, starvation and miserable conditions), people had to abandon their rural location to settle in the hastily constructed shanty towns at the periphery of large cities. The new migrants often bring dogs with them and due to its close relationship with humans, the domestic dog the main reservoir of VL transmit VL in many countries. Similar situation could have existed in Bangladesh, where cattle, sheep, goat, dogs and domestic cats are found to live in a common habitant and allowing free living vector sandflies to bite and suck blood. Moreover, the open border of Bangladesh could have allowed easy migration of people from neighbouring country and may contribute transmission of diseases. Jackal in Bangladesh has emerged as a new carrier of VL, as village condition provided an excellent habitat for them, the jackals move readily from one place to others for searching of food, may easily transmit the disease to other animals and humans (Khan et al., 2012).

In 1960s intensive vector control measures were adapted under the Malaria Eradication Program in this subcontinent and VL had almost been eliminated from this part of the world, illustrating the vulnerability of the transmission cycle. Before 1977-78, due to the absence of an effective surveillance system in Bihar and other affected areas, only limited data were available about cases of Kala-azar. The VL turned endemic since 1960 and in every 10-15 years the disease become epidemic in the endemic regions of this subcontinent. The goal of eliminating Kala-azar from India by 2010 was lost despite paramount efforts taken by the Government of India and World Health Organization and Regional Office for South East Asia. The possible reason for the failure of Kala-azar elimination program were due to changes in the vector control practices (lack of massive vector control programme), therapeutic modalities used against VL or unidentified vector or carrier animals spreading the diseases. Therapeutic modalities were found to be highly correlating with VL mortality not with VL morbidity. The diminishing efficacy of first and second line drugs and the introduction of new drugs and drugs combination were responsible for ups and downs in the VL mortality. The VL mortality is constantly declining since 1993, but cases started increasing from 2003 to 2007 and then rose again during 2010 to 2011. This showed a serious lacuna in the combined efford of human and veterinary researchers and lack of nation wide effective vector control practices. Since the independence of Bangladesh massive vector control/ eradication programme virtually was not carried out and the VL becoming more endemic to epidemic in Bangladesh. It is concluded that, the vector control practices have direct impact on the incidence of VL while, the treatment modalities evolved in response to the development of drug resistance have direct impact on case fatality (Mondal et al., 2008). After recovery following treatment, some patients develop PKDL or after treatment about $67-70 \%$ of the patients with VL and immunosupressive diseases may get relapse and may act as reservoir for leishmania parasite and would maintain the transmission cycle and facilitate transmission during inter-outbreak periods. Moreover, wild canids (Fig. 3b), rodents, ruminants (Fig. 3a) and few other unidentified carrier animals may transmit the disease (Khan et al., 2012; Muniaraj et al., 2014) and require constant monitoring on to the occurance or carrier nature of leishmaniasis.

Malaria: After leishmania, malaria is an important zoonotic and vector- borne parasitic pathogens transmitted by infected Anopheles mosquitoes, which bite mammalian hosts mainly between dusk and dawn. Globally there are more than 60 species of anophelines which are recognized as bological vectors. Malaria is caused by four species of parasites in humans: Plasmodium falciparum, $P$. vivax, $P$. malariae and $P$. ovale. Malaria transmission occurs in all six WHO regions. Globally, an estimated 3.2 billion people in 97 countries and territories are at risk of being infected with malaria and developing disease and 1.2 billion people are at high risk. According to the latest estimates (http://www.who.int/gho/malaria/epidemic/en/), 198 million cases of malaria occurred globally in 2013 (uncertainty range 124-283 million) and the disease led to 584000 deaths (uncertainty range 367000-755000). About 207 million cases were recorded in 2012 with 627000 deaths. The burden was heaviest in the WHO African region, where an estimated $90 \%$ of all malarial deaths occur. South-east Asia accounts for $9 \%$ of the total burden and Bangladesh is considered as one of the malaria endemic countries in South Asia. Children aged under 


\section{A. H. N. A. Khan}

five years had death rate of about $78 \%$ of all deaths. Poor and vulnerable communities living in rural areas with limited access to health facilities suffer the most. Four out of 10 people who die of malaria live in the 2 highest burden countries: the Democratic Republic of the Congo and Nigeria. In the Amazon and East Africa, there is increase in standing water and sunlight due to deforestation enhances breeding success of some mosquito species, which may increase the risk of malaria. Further increases in urbanization frequently eliminate Anopheline mosquitos' habitat and could have reduced malaria elsewhere. General symptoms of malaria include headache, nausea, fever, vomiting and flu-like symptoms, however, these can vary depending on the species causing the infection. Bangladesh has 34 Anopheles mosquitoe species. An entomological investigation conducted by ICDDR,B scientists identified seven species of mosquitoes transmitting malaria in human included Anopheles Karwari, An. maculatus, An. barbriostris, An. nigerrimus, An. vagus, An. subpictus and An. Philippinensis. Following insect bites malaria symptoms appears after a period of seven days or longer. If untreated, the disease can lead severe complications and death. Malaria caused by P. falciparum (also called malignant or falciparum malaria) is the most virulent form of malaria, with the highest rates of complications and mortality (Rich et al., 2009). According to WHO guidelines, the choice of artemisinin-based combination therapies could be artemether plus lumefantrine or artesunate plus amodiaquine or artesunate plus mefloquine or artesunate plus sulfadoxine-pyrimethamine or dihydroartemisinin plus piperaquine (WHO, 2010). The best way to prevent malaria infection is through the regular use of long-lasting insecticidal nets, spraying of homes with residual insecticides, and the use of WHO-recommended curative and preventive therapies.

African trypanosomiasis: African trypanosomiasis (sleeping sickness) is an illness endemic to sub-Saharan Africa and limited to the continent of Africa where its insect vector, the tsetse fly, is found. It is caused by 2 subspecies of the flagellate protozoan Trypanosoma brucei. There are two forms of sleeping sickness. The chronic gambiense form is found in Central and West Africa and although an animal can be found infected, the disease is maintained by transmission between the insect vector and humans. However, the animal reservoir is important in the acute rhodesiense form found in Eastern and Southern Africa. The causal agent, Trypanosoma brucei rhodesiense, infects humans, wild animals and domestic livestock, which maintain infection between epidemics, and coexists in animals with a complex of pathogenic trypanosomes (T. congolense, T. vivax and T. $b$. brucei) that present a major problem for livestock keepers in Africa. Untreated, the disease is always fatal in humans and treatment is expensive, normally costs from US\$150 to US\$800 per person. In the later stages of the disease treatment itself involves about 5\% mortality. Control is via the vector or the disease's human and livestock reservoirs (Giordani et al., 2014). For rhodesiense, the key to preventing the disease in people is now thought to be by treating the cattle reservoir, using drugs which are effective not only against the trypanosomes pathogenic to humans but also those which cause substantial losses to livestock production; backed up by appropriate vector control measures.

Dengue: Dengue is the most rapidly spreading mosquito-borne viral disease in the world. Unlike other mosquitoes, Aedes aegypti, the main vector for dengue bites during the day. Aedes albopictus, a secondary dengue vector, can survive in cooler temperate regions. There are 4 closely related serotypes of the virus that cause dengue and the lifelong immunity developed after infection. In the past 50 years, incidence of dengue has increased 30-fold with increasing geographical expansion to new countries and, in the present decade, from urban to rural settings. More than 2.5 billion people - over $40 \%$ of the world's population - are now at risk from dengue. WHO currently estimated that, there are about 50-100 million dengue infections worldwide every year. The disease is endemic in South East Asian country including Bangladesh, India, Indonesia, Maldives, Myanmar, Sri Lanka, Thailand, Bhutan, Korea and Timor-Leste. In USA the disease is more frequent in Barbados, Colombia, Dominican Republic, El Salvador, Guatemala, French Guyana, Mexico, Peru, Puerto Rico and Venezuela.

Severe dengue also known as dengue haemorrhagic fever (Fig. 4c) is found in tropical and sub-tropical locations in most Asian and Latin American countries. An estimated 500000 people with severe dengue require hospitalization each year, a large proportion of these are children. About $2.5 \%$ of those affected die. Symptoms of dengue include fever, severe headache, pain behind the eyes, muscle and joint pain, swollen glands and rash. There is no vaccine or any specific medicine to treat dengue. People who have dengue fever should rest, drink plenty of fluids and reduce the fever using paracetamol. Severe dengue (also known as dengue hemorrhagic 
fever) is characterized by fever, abdominal pain, persistent vomiting, bleeding and difficulty breathing. It is a potentially lethal complication, affecting mainly children. The only method to reduce transmission of dengue virus is to control vector mosquitoes and protect human and animals from mosquito's bites. Severe dengue is associated with severe bleeding, severe organ dysfunction, or severe plasma leakage while all other cases are uncomplicated (Clark, 2005). The 1997 classification divided dengue into undifferentiated fever, dengue fever, and dengue hemorrhagic fever. Dengue hemorrhagic fever was subdivided further into grades I-IV. Grade I is the presence only of easy bruising or a positive tourniquet test in someone with fever, grade II is the presence of spontaneous bleeding into the skin and elsewhere, grade III is the clinical evidence of shock, and grade IV is shock so severe that blood pressure and pulse cannot be detected. Grades III and IV are referred to as "dengue shock syndrome" (Dalrymple and Mackow, 2012). There are no specific antiviral drugs for dengue, however maintaining proper fluid balance is important. The life threatening cases require extensive respiratory, cardiac and platelets therapy. However, the case report of human dengue in Bangladesh although was about 5000 to 6000 each year at around year 2000 (Rahman et al., 2002), the incidence has been reduced by now and may be due to awareness of people living in dengue endemic zone (mostly Dhaka) and dry up their habitants.

Nipah virus infection: Nipah virus (NiV) infection is a newly emerging zoonosis that causes severe disease both in man and animals. The natural host of the viruses is fruit bats of the Pteropodidae Family, Pteropus genus. NiV was first identified during an outbreak of disease that took place in Kampung Sungai Nipah, Malaysia in 1998. On this occasion, pigs were the intermediate hosts. However, in subsequent NiV outbreaks, there were no intermediate hosts. In Bangladesh during 2004, humans became infected with $\mathrm{NiV}$ as a result of consuming date palm sap that had been contaminated by infected fruit bats. Human-to-human transmission has also been documented, including in a hospital setting in India. NiV infection in humans has a range of clinical presentations, from asymptomatic infection to acute respiratory syndrome and fatal encephalitis. NiV is also capable of causing disease in pigs and other domestic animals. Because of the danger of human infection from infected pigs and the emergency setting, clinical observations were not detailed in the field during the original epidemic. Most pigs developed a febrile respiratory disease with a severe cough that led to the local names for the disease-"barking pig syndrome" and "one-mile cough." Encephalitis was also noted, particularly in the sows and boars in affected facilities. There is no vaccine for either humans or animals. The primary treatment for human cases is symptomatic and requires intensive supportive care.

Ebola: Ebola, previously known as Ebola hemorrhagic fever, is a rare and deadly occurring disease caused by infection with one of the Ebola virus strains. Ebola can cause disease in humans, monkeys, gorillas, and chimpanzees. There are five identified Ebola virus species, four of which are known to cause disease in humans: Ebola virus (Zaire ebolavirus); Sudan virus (Sudan ebolavirus); Tai Forest virus (Tai Forest ebolavirus, formerly Cote d'Ivoire ebolavirus); and Bundibugyo virus (Bundibugyo ebolavirus). The fifth, Reston virus (Reston ebolavirus), has caused disease in nonhuman primates, but not in humans. Ebola was first discovered in 1976 near the Ebola River in what is now the Democratic Republic of the Congo. Since then, outbreaks have appeared sporadically in Africa. The disease has been reported in Africa, USA, UK, France, Germany, Italy, India, Norway, Ireland, Switzerland and Democratic Republic of the Congo.

It is not entirely clear how an Ebola outbreak starts (Gonzalez et al., 2007). The initial infection is believed to occur after an Ebola virus is transmitted to a human by contact with an infected animal's body fluids. Evidence strongly implicates bats act as the reservoir hosts for ebolaviruses. Bats drop partially eaten fruits and pulp, then land mammals such as gorillas and duikers feed on these fallen fruits. This chain of events forms a possible indirect means of transmission from the natural host to animal populations (CDC, 2014). However, on the basis of evidence and the nature of similar viruses, researchers believe that the virus is animal-borne and bats are the most likely reservoir. Ebola enters in to the body through mouth, nose and eyes, or a break in the skin. Ebola causes sudden high fever, extreme tiredness, headache, body pain, loss of appetite and abdominal pain. This is often followed by severe vomiting and diarrhoea. Some patients may experience the loss of blood through bleeding internally and/or externally. The average case fatality rate is varied from $25 \%$ to $90 \%$. No proven Ebola virus-specific treatment presently exists. Fluid replacement to regain extreme loss of fluids may prevent hypovolaemic shock. It needs to avoid eating or shearing bat eaten fruits from any source to prevent Ebola. 
Rabies: Bats are increasingly implicated as an important wildlife reservoirs for variants of rabies virus transmitted to humans. All mammals can contract and carry rabies; however bats are not asymptomatic carriers of the disease. In reality, bats contract rabies far less than other animals. Less than $1 / 2$ of $1 \%$ of all bats may contract the disease. A variety of wild animals (rabies vector species) can catch rabies, including jackals, foxes, skunks, raccoons, coyotes and bats. Cats and dogs and even livestock can contract rabies. There is only one to two human deaths per year from bat rabies in the United States. A person living in the U.S. is more likely to catch leprosy or the plague than to contract rabies from a bat. Throughout the world $30,000+$ people die from the disease each year - $99 \%$ of these deaths come from contact with rabid dogs. Rabies is mostly transmitted through a bite. Although rare, exposures can also occur from contact between infected saliva or nervous tissues and open wounds or the mucous membranes of the eyes, nose, or mouth. The principal source of rabies exposure from bats is through careless handling and negligencies following bite of bats. Keeping domestic pets vaccinated against rabies is critical in rabies prevention. Understanding how to peacefully co-exist with bats, teaching children to never handle bats and never attempting to rescue a bat bare-handed will minimize the risk of contracting the disease. Ninety to $95 \%$ of sick bats are not rabid, but taking a careless chance on being bitten could prove fatal.

Any animal bite (domestic or wild) should be reported immediately to a family physician or public health professional for evaluation as a possible rabies exposure. Vaccines are no longer administered in the abdomen now a day. Vaccines available are human diploid cell culture vaccine and typically administered in the upper-arm or thigh. The injections are relatively painless, like a flu shot or a tetanus shot and $100 \%$ protective.

Plague: Plague is a deadly infectious disease that is caused by the enterobacteria Yersinia pestis, named after the French-Swiss bacteriologist Alexandre Yersin. Until June 2007, plague was one of the three epidemic diseases specifically reportable to the World Health Organization (the other two being cholera and yellow fever). The oriental rat flea (Xenopsylla cheopsis) is the primary vector for transmitting Yersinia pestis in most plague epidemics in Asia, Africa, and South America. Both male and female fleas feed on blood of man and rats and transmit the infection. Depending on lung infection, or sanitary conditions, plague can also be spread in the air, by direct contact, or by contaminated undercooked food or materials. The symptoms of plague depend on the concentrated areas of infection in each person: a) bubonic plague affect lymph nodes, b) septicemic plague in blood vessels and c) pneumonic plague in lungs. It is treatable if detected early. Plague is still endemic in some parts of the world and curative.

Typhoid fever: Typhoid fever is a life-threatening illness caused by the bacterium Salmonella typhi. Most cases (up to $75 \%$ ) are acquired while traveling internationally. Typhoid fever is still common in the developing world, where it affects about 21.5 million people each year. S. typhi lives mostly in humans. Persons with typhoid fever carry the bacteria in their bloodstream and intestinal tract. In addition, a small number of persons, called carriers, recover from typhoid fever but continue to carry the bacteria. Both ill persons and carriers shed Salmonella Typhi in their feces (stool). The most common way of transmitting the bacteria is through contaminated feed and drinks. The flies can transmit typhoid fever mechanically in unhygienic environments especially through food and drink. In the period of 10 to 15 days incubation, disorders are seen overall, a phase of bacteremia with fever, which increases progressively up to $39-40^{\circ} \mathrm{C}$, at which point remains, headache, stupor, rosella in the belly, swelling of the nasal mucosa, roasted tongue, ulceration of the palate and, sometimes, hepato-splenomegaly, and diarrhea. Sanitation and hygiene are the crucial to prevent transmission of typhoid fever. Carriers of the bacteria may not work as food handlers.

Chikungunya: Chikungunya is a viral tropical disease transmitted also by Aedes mosquitoes. It is relatively uncommon and poorly documented. The disease has been found in Africa, Asia, and on islands in the Caribbean, Indian and Pacific Oceans. Typical symptoms are an acute illness with fever, skin rash and incapacitating joint pains that can last for weeks. The latter distinguishes chikungunya virus from dengue, which otherwise shares the same vectors, symptoms and geographical distribution. There is no cure or commercial vaccine for the disease. Most patients recover fully but, in some cases, joint pain may persist for several months or even years. As with dengue, the only method to reduce transmission of chikungunya virus is to control vector mosquitoes and protect against mosquitoes bites. 
Lyme disease: Lyme disease (Lyme borreliosis) is caused by Borrelia bacteria and is transmitted through the bite of infected deer ticks Ixodes species. Many species of mammals can be infected and rodents and deer act as important reservoirs. The first recognized outbreak of this disease occurred in Connecticut, United States, in 1975. The current burden is estimated at 7.9 cases per 100000 people in the United States, according to the US Centers for Disease Control and Prevention. Since the mid-1980s, the disease began to be reported in several European countries. Lyme disease occurs in rural areas of Asia, north-western, central and eastern Europe, and the United States of America. It is now the most common tick-borne disease in the Northern Hemisphere. People living in or visiting rural areas, particularly campers and hikers, are most at risk. The symptoms of Lyme disease include fever, chills, headache, fatigue, muscle and joint pain. A red ring or bull's-eye rash (Fig. 4b) often appears at the site of the tick bite and gradually expands to a ring with a central clear zone, before spreading to other parts of the body. If left untreated, infection can spread to joints, the heart and central nervous system. Arthritis may develop up to 2 years after onset. Most cases of Lyme disease can be treated successfully with a course of antibiotics.

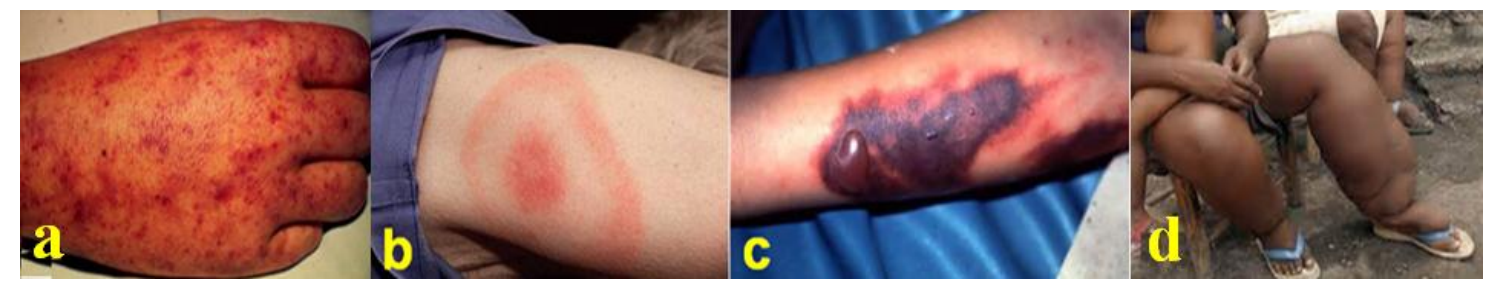

Fig. 4. Lesions and pathology as seen in the hand of human due to rocky mountain spotted fever (a), Lyme disease (b), Hemorrhagic dengue (c) and Elephantiasis (d, legs). Sources (www.cdc.gov).

Yellow fever: Yellow fever is an acute viral haemorrhagic disease transmitted by Aedes mosquitoes. The "yellow" in the name refers to the jaundice that affects some patients. There are an estimated 200000 cases of yellow fever causing 30000 deaths worldwide each year. The virus that causes yellow fever is endemic in tropical areas of Africa and Latin America where a combined population of over 900 million people lives. Small numbers of imported cases occur in countries free of yellow fever. Symptoms of the disease include fever, muscle pain with prominent backache, headache, shivers, loss of appetite, and nausea or vomiting. Most patients improve and their symptoms disappear after 3 to 4 days. However, $15 \%$ of patients enter a second, more toxic phase within 24 hours of the initial remission. High fever returns and several body systems are affected. The patient rapidly develops jaundice and complains of abdominal pain with vomiting and internal bleeding. Half of these patients die within 10 to 14 days. There is no specific treatment for yellow fever, only supportive care to treat dehydration, respiratory failure and fever. Vaccination is the most important preventive measure against yellow fever. The vaccine is safe, affordable and highly effective. A single dose of yellow fever vaccine is sufficient to provide life-long protection against the disease.

Japanese encephalitis: Japanese encephalitis virus is transmitted to humans through infected Culex mosquitoes. Japanese encephalitis causes an estimated 50000 cases and 10000 deaths every year, mostly of children aged less than 5. It occurs across Asia, from the islands of the Western Pacific in the east to the Pakistani border in the west and from Korea in the north to Papua New Guinea in the south. Most human infections are asymptomatic or result in only mild symptoms. However, a small percentage of infected people develop inflammation of the brain (encephalitis), with symptoms including the sudden onset of headache, high fever, disorientation, coma, tremors and convulsions. One quarter of severe cases can be fatal and $30 \%$ of those who survive severe infection have lasting central nervous system damage. Transmission of the Japanese encephalitis virus occurs primarily in rural agricultural areas, particularly around flooded rice fields, but can also occur near urban centers. In temperate areas of Asia, transmission of the virus is seasonal. The virus is transmitted between mosquitoes, in particular Culex tritaeniorhynchus, and animals such as pigs and wading birds. Humans are incidental or "dead-end" hosts, because they usually do not develop high-enough concentrations of the virus in their bloodstreams to infect feeding mosquitoes. There is no specific treatment for the disease; supportive care and management of 
complications can provide some relief. The vaccine against Japanese encephalitis is the single most effective preventive measure against this disease.

Lymphatic filariasis: Infection with lymphatic filariasis, commonly known as elephantiasis, occurs when thread-like, filarial parasites are transmitted to humans through mosquitoes. Lymphatic filariasis is transmitted by different types of mosquitoes, for example by the Culex mosquito, widespread across urban and semi-urban areas; Anopheles, mainly in rural areas; and Aedes, mainly in the Pacific Islands and parts of the Philippines. It is also transmitted by 3 types of parasite (Wuchereria bancrofti, responsible for $90 \%$ of cases, Brugia malayi and B. timori). Microscopic parasitic worms lodge in the lymphatic system and disrupt the immune system. They live for 6-8 years and, during their lifetime, produce millions of microfilariae (tiny larvae) that circulate in the blood. More than 120 million people are currently infected with lymphatic filariasis, about 40 million of them are disfigured and incapacitated by the disease. Lymphatic filariasis afflicts more than 25 million men with genital disease and more than 15 million people with lymphoedema. The majority of infections have no symptoms but silently cause damage to the lymphatic system and the kidneys as well as alter the body's immune system. Acute episodes of local inflammation involving skin, lymph nodes and lymphatic vessels often accompany chronic lymphoedema (Fig. 4d). Approximately $65 \%$ of those infected live in the WHO South-East Asia Region, 30\% in the African Region, and the rest in other tropical areas. Treatment is rarely recommended to clear the adult parasites from the bloodstream by using a single dose of albendazole together with either diethylcarbamazine or ivermectin. Interruption of transmission of infection by mosquitoes bite is mostly recommended.

Dirofilariasis (heartworms): Heartworms is caused by the nematode (roundworm) Dirofilaria immitis, a parasitic disease of canids, felids and human and is transmitted by the bite of mosquitoes. The severity of this disease is directly dependent upon the number of worms present in right ventricle of heart (Fig. 5) and pulmonary artery, the duration of the infestation, and the response of the host (the infested dog is the host). Adult heartworms produce hundreds of thousands of microscopic offspring known as microfilaria that circulate in the infected animal's bloodstream. They don't develop further until they are ingested by mosquito. When a mosquito takes blood, it also takes in microfilaria that will develop into heartworm larvae inside the mosquito's gut over a span of around two weeks. The larvae congregate around the mosquito's mouthparts. While the infected mosquitoes suck blood mostly at early night transmit the immature worm in to the host. These larvae migrate from the bite wound through the dog's body until they reach the heart and blood vessels of the lungs, a process that takes approximately six months. The larvae mature in the dog's body an adult heartworm can grow to be about 12 inches long. The heartworm infestation in dogs and jackals is defined in three classes depending upon the varying in severity. Dogs with Class I heartworm disease are often asymptomatic. Class II patients usually exhibit coughing and unusual intolerance to exercise. The most severe cases, defined as Class III, showed symptoms of anemia, exercise intolerance, fainting spells, unproductive coughing and - in severely affected dogs, right-sided chronic heart failure.

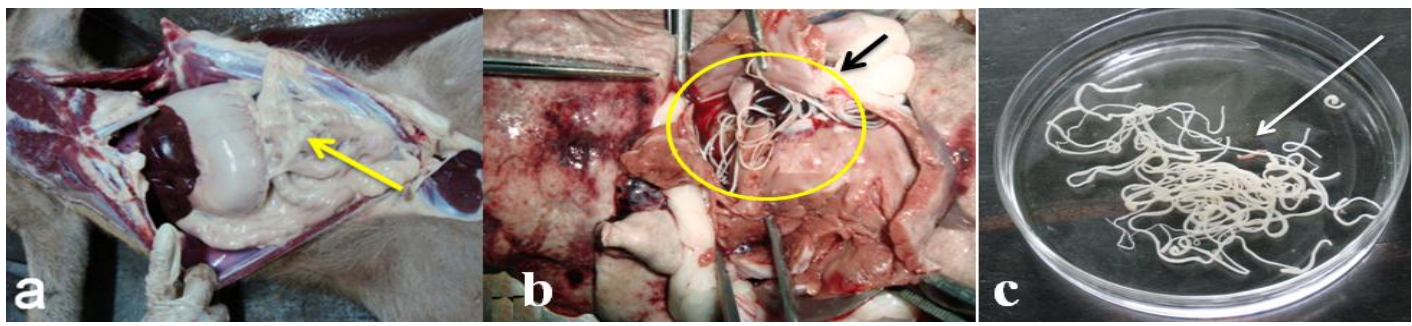

Fig. 5. Out of 15 jackals examined (a), 13 jackals of BAU campus found to carry heartworm (H) in their right ventricles and pulmonary artery ( $b$ and c). Peripheral blood collected at night and stained with Giemsa showed the presence of microfilaria in the blood. Street dogs collected from the BAU campus also found to carry heartworm in their right ventricle, BAU campus was, therefore, acted as an endemic zone of heartworm infestation. 
The heartworm is mainly endemic in geographic areas with tropical and subtropical climates, and is also commonly found along the Atlantic and Gulf Coasts, and the Ohio and Mississippi river basins. Dog heartworm is common in the dogs and jackals at Bangladesh Agricultural University campus and most of the canids have been carrying heartworm. The situation could have exists in the canids of other region of Bangladesh. In regions where $D$. immitis is endemic, dogs without proper heartworm protection are almost 100 percent likely to suffer from heartworm infestation. Human being are infected with the parasite. The skin affected more following bites of infected mosquitoes, the parasite infrequently may pass upon the systemic circulation but trapped in the pulmonic vessels where it produce nodular lesions like that of typical cases of tuberculosis. The adult worm saldom seen in the heart of human being. Heartworm disease is preventable with the administration of a heartworm prophylaxis (preventative) medication, as recommended by OIE. For those dogs that do contract heartworm disease, the prognosis is good for mild to moderate cases, and such conditions can be relatively uneventful. Dogs with more severe cases may suffer from lung complications and anthelmentic therapy is not recommended as the dead parasite could have developed emboli with fatal consequences. Detection of microfileria from the peripheral blood collected at night is the key to diagnose the disease. Risk factors associated with heartworm infestation in humans include residence in endemic regions, such as tropical areas, outdoor habitation, and lack of the proper prophylaxis to prevent heartworm infestation.

Q fever: Q fever was first recognized as a human disease in Australia in 1935 and in the United States in the early 1940's. The "Q" stands for "query" and was applied at a time when the causative agent was unknown. Q fever is a worldwide disease with acute and chronic stages caused by the bacteria Coxiella burnetii. Cattle, sheep, and goats are the primary reservoirs although a variety of species may be infected. Organisms are excreted in milk, urine, and feces of infected animals. During birthing the organisms are shed in high numbers within the amniotic fluids and the placenta. The organism is extremely hardy and resistant to heat, drying, and many common disinfectants which enable the bacteria to survive for long periods in the environment. Infection of humans usually occurs by inhalation of these organisms from air that contains airborne barnyard dust contaminated by dried placental material, birth fluids, and excreta of infected animals. Other modes of transmission to humans, including tick bites, ingestion of unpasteurized milk or dairy products, human to human transmission is rare. Humans are often very susceptible to the disease, and very few organisms may be required to cause infection. The traffic of infected ruminants may be one of the most important sources of transmitting infection.

The symptom in humans are high fevers (up to $104-105^{\circ} \mathrm{F}$ ), severe headache, general malaise, myalgia, chills and/or sweats, non-productive cough, nausea, vomiting, diarrhea, abdominal pain and very often chest pain. Although most persons with acute $\mathrm{Q}$ fever infection recover, others may experience serious illness with complications that may include pneumonia, granulomatous hepatitis (inflammation of the liver), myocarditis (inflammation of the heart tissue) and central nervous system complications. Pregnant women who are infected may be at risk for pre-term delivery or miscarriage.

Rocky Mountain spotted fever (RMSF): Rocky Mountain spotted fever is the most lethal and frequently reported rickettsial illness in the United States. It has been diagnosed throughout the Americas. Rocky Mountain spotted fever in other countries popularly known as "tick typhus," "Tobia fever" "Sao Paulo fever" or "febre maculosa" (Brazil), and fiebre manchada (Mexico). The disease is caused by Rickettsia rickettsii, a species of bacterium that is spread to humans by Dermacentor ticks. Initial signs and symptoms of the disease include sudden onset of fever, headache, and muscle pain, followed by development of rash (Fig. 4a). The disease can be difficult to diagnose in the early stages, and without prompt and appropriate treatment it can be fatal. Patients who had a particularly severe infection requiring prolonged hospitalization and long-term health problems may caused by this disease. Rickettsia rickettsii infects the endothelial cells that line the blood vessels. The damage that occurs in the blood vessels results in a disease process called a "vasculitis", and bleeding or clotting in the brain or other vital organs may occur. Loss of fluid from damaged vessels can result in loss of circulation to the extremities and damaged fingers, toes or even limbs may ultimately need to be amputated. Patients who suffer this kind of severe vasculitis in the first two weeks of illness may also be left with permanent long-term health problems such as profound neurological deficits, or damage to internal organs. Those who do not have this kind of vascular damage in the initial stages of the disease typically recover fully within several days to months. 
Babesiosis: Babesiosis is caused by protozoan parasite, transmitted by vector tick and the parasites are not transmitted from person-to-person like the flu or the common cold. Babesia microti, B. divergens and B. duncani is transmitted to human by Ixodes scapularis ticks (also called blacklegged ticks or deer ticks). Many different species (types) of Babesia parasites have been found in animals, only a few of which have been found in people. $B$. microti, which usually infects white-footed mice and other small mammals, is the main species that has been found in people in the United States. However, human to human transmission is well recognized to occur through blood transfusions. Most infections are probably asymptomatic, as indicated by serologic surveys. Manifestations of disease include fever, chills, sweating, myalgias, fatigue, hepatosplenomegaly, and hemolytic anemia. Symptoms typically occur after an incubation period of 1 to 4 weeks, and can last several weeks. The disease is more severe in patients who are immunosuppressed, splenectomized, and/or elderly. Occasional (sporadic) cases of babesiosis caused by other Babesia species have been detected but not fatal.

Crimean-Congo haemorrhagic fever: Crimean-Congo haemorrhagic fever is a tick-borne viral disease that kills $30 \%$ of infected people. The virus is transmitted to people either from tick-bites (Hyalomma spp.) or through contact with blood or tissues of infected animals such as cattle, sheep, goats and ostriches. Human-tohuman transmission can occur resulting from close contact with the blood, organs or other bodily fluids of infected persons. The virus occurs in Africa, the Balkans and Asia. Onset of symptoms is sudden, with fever, muscle ache, dizziness, neck pain and stiffness, backache, headache, sore eyes and sensitivity to light. There may be nausea, vomiting, diarrhea, abdominal pain and sore throat early on, followed by sharp mood swings and confusion. Other signs include a faster heart rate, a rash, enlarged lymph nodes and liver and kidney failure. More than one third of those infected die within the second week of illness. The main approach to treating Crimean-Congo haemorrhagic fever is treating symptoms. The antiviral drug ribavirin has been shown to be effective. There is currently no safe and effective vaccine widely available for human use. The best way to reduce infection in people is to control tick infestations and prevent tick-bites. People handling animals during slaughtering or butchering should wear protective clothing and the animals should be treated with pesticides two weeks before the slaughter.

Schistosomiasis: Schistosomiasis is a chronic, parasitic disease caused by blood flukes (trematode worms). People become infected when larval forms of the parasite - released cercaria by freshwater snails - penetrate the skin during contact with infested water. In the body, the larvae develop into adult schistosomes. Adult worms live in the blood vessels where the females release eggs. Some of the eggs are passed out of the body in the faeces or urine to continue the parasite life-cycle. Others become trapped in body tissues, causing an immune reaction and progressive damage to organs and blood vessels. In 2012, more than 42.1 million people were treated for schistosomiasis. Schistosomiasis is found in 78 countries in tropical and sub-tropical areas, mostly in Africa. People are at risk of infection due to agricultural, domestic and recreational activities which expose them to infested water.

Humans are the reservoir for S. mansoni and S. haematobium. Cattle, water buffalo, horses, dogs, cats, rodents and monkeys are commonly infected with $S$. japonicum. Intermediate hosts are species of snails (Biomphalaria and Bulinus). S. mansoni occurs in Africa, South America and some Caribbean islands (including Puerto Rico); S. haematobium in Africa and the Middle East; and S. japonicum in China, Japan, the Philippines, and South East Asia. Schistosoma haematobium is an important digenetic trematode, and is found in urinary system of man in Africa and the Middle East. Require to take special precaution not to swim and walk in water resources containing cercaria of schistosomes.

Symptoms of schistosomiasis are caused by the body's reaction to the worms' eggs, and not by the worms themselves. Intestinal schistosomiasis can cause abdominal pain, diarrhea, blood in the stool, enlargement of liver and spleen, fluid in the peritoneal cavity and hypertension of the abdominal blood vessels. Urogenital schistosomiasis causes blood in the urine, lesions and fibrosis of the bladder, ureter and genitals, kidney damage and sometimes bladder cancer. It can cause infertility. In children, schistosomiasis can cause anaemia and stunting, reducing their learning abilities. The control of schistosomiasis is based on regular, large-scale treatment of at-risk population groups, access to safe water, improved sanitation, hygiene education and snail control. 
Vector-borne diseases with zoonotic potential

\section{Conclusions and recommendations}

The increase incidence of VBDs is related to the availability of vectors, pathogens and transmission to the definitive hosts. Changes in environmental temperature directly affect emergence and transmission of VBPs through pathogen-host interaction, and indirectly through changes in ecosystem and species composition. As temperatures increases in some geographical areas, the vectors have been spreading to areas were previously was too cold. For example, two mosquito vectors that carry malaria in Asia are now available in the USA-Mexico border. Leishmanial vector are now available in USA and few of the European country. More frequent droughts in some areas can cause a decrease in vectors densities that require water for their life cycle. A decline in biodiversity alters predator-prey relationships; a decline in the predators of vectors can increase vector populations. Movement of human population and deforestation can also expand distribution of pathogens and increase exposure routes.

Collectively, arthropods are responsible for millions of illness in man and animals each year. Over the past 30 years, there has been a global emergence and re-emergence of infectious disease in man and animals and vectorborne diseases in particularly showed an increased in frequency of epidemic transmission and crossing of geographic barrier. A major problem is that the most important vector-borne diseases occurred in the tropics, usually in the areas where resources are limited and surveillance is poor. However, the shrinking world, with highly increased human and animal mobility due to air travel and commerce (globalization) has made these diseases not just problems of the tropics; they present the global community with possibly its greatest health problem and threat to economic security today. This underscores the need for physicians, veterinarians and ecobiologist in endemic and non-endemic areas to be aware of vector-borne diseases and to be knowledgeable about where they occur and how to recognize and treat them. There is strong need to set rules that prevent emission of greenhouse gases that influence local/ regional ecology, alter the life cycles of certain diseases in vectors and animals. It is mandatory to preserve $20-25 \%$ forests areas and wetlands to maintain healthy ecology and keep vectors in forest; now a day this requirement is greatly ignored. Developing and implementing early warning systems to reduce exposure to environmental hazards and limit susceptibility in exposed populations is also lacking. It needs to develop expert manpower, deploy innovative disease prevention techniques and work to improve diagnostic testing for vector-borne diseases. Development of new pesticides is needed aimed at controlling disease vectors with species specificity (affecting only the target vectors), environmentally safe and low susceptibility to resistance. Routine monitoring in the genetics of vector-borne pathogens also needed to understand the changes in the genomes with their changing ecology and host tropism. The registered vet equipped to deal with a number of insect borne infectious and zoonotic diseases need to deploy in the cross border areas, hilly areas, low land, marshy land, airport and forest areas of a country to keep constant monitoring of the existing or emerging vectors and vector-borne diseases and design preventive and control strategies accordingly.

\section{REFERENCES}

1. Bailey MS and Lockwood DN (2007). Cutaneous leishmaniasis. Clinical Dermatology 25: 203-211.

2. CDC (2014). Ebola Hemorrhagic Fever Preventio. Retrieved 2014-08-02. http://www.cdc.gov/vhf/ebola/prevention/index.html

3. Clark DV (2005). Economic impact of dengue fever/dengue hemorrhagic fever in Thailand at the family and population levels. American Journal of Tropical Medicine and Hygiene 72: 786-791.

4. Dalrymple NA and Mackow ER (2012). Roles for Endothelial Cells in Dengue Virus Infection. Review Article. Advances in Virology Article ID 840654: 1-8.

5. Giordani F, Mwenechanya R and Barrett MP (2014). Chapter 39-Advances in Understanding and Treatment of Human African Trypanosomiasis: Divergent Diseases Caused by Distinct Parasites. Handbook of Pharmacogenomics and Stratified Medicine 901-917.

6. Gonzalez JP, Pourrut X and Leroy E (2007). Ebolavirus and other filoviruses. Current Topics in Microbiology and Immunology 315: 363-387.

7. Gonzalez U, Pinart M, Rengifo-Pardom M, Macayam A, Alvarm J and Tweed JA (2009). Interventions for American cutaneous and mucocutaneous leishmaniasis (Review). The Cochrane Collaboration 2: 1-42.

8. Gubler DJ (2009). Vector-borne diseases. Scientific and Technical Review of the Office International des Epizooties 28: 583-588. 
9. Khan MAHNA, Khanm SS, Bashu J, Rima UK, Pervin M, Hossain MZ, Habib MA, Chowdhury GA and Hossain MM (2012). Visceral leishmaniasis is endemic in Golden Jackals of Bangladesh Agricultural University Campus, a threat for expanding future zoonotic visceral leishmaniasis. Bangladesh Journal of Veterinary Medicine 10: 97-105.

10. Khanjani N, Gonzalez U, Leonardi-Bee J, Mohebali M, Saffari M and Khamesipour A (2009). Vaccines for preventing cutaneous leishmaniasis (protocol). The Cochrane Collaboration 2: 1-12.

11. Mondal D, Alam MS, Karim Z, Haque R, Boelaert M and Kroeger A (2008). Present situation of vector-control management in Bangladesh: A wake up call. Health Policy 87: 369-376.

12. Muniaraj M (2014). The lost hope of elimination of Kala-azar (visceral leishmaniasis) by 2010 and cyclic occurrence of its outbreak in India, blame falls on vector control practices or co-infection with human immunodeficiency virus or therapeutic modalities? Tropical Parasitology 4: 10-19.

13. Rahman M, Rahman K and Siddque AK (2002). First outbreak of dengue hemorrhagic fever, Bangladesh. Emerging Infectious Diseases CDC 8.

14. Rich SM, Leendertz FH, Xu G, Lebreton M, Djoko CF, Aminake MN, Takang EE, Diffo JLD, Pike BL, Rosenthal BM, Formenty P, Boesch C, Ayala FJ and Wolfe ND (2009). "The origin of malignant malaria". Proceedings of the National Academy of Sciences 106: 14902-14907.

15. Samad MA (2011). Public health threat caused by zoonotic diseases in Bangladesh. Bangladesh Journal of Veterinary Medicine 9: 95-120.

16. Torres DF (2006). Leishmanial vaccine: the newest tool for prevention and control of canine visceral leishmaniasis and its potential as a transmission-blocking vaccine. Veterinary Parasitology 141: 1-8.

17. Torres DF (2007). The role of dogs as reservoirs of Leishmania parasites, with emphasis on Leishmania (Leishmania) infantum and Leishmania (Viannia) braziliensis. Veterinary Parasitology 149: 139-146.

18. WHO (2010). Treatment of severe P. falciparum malaria. In: Guidelines for the treatment of malaria. $2^{\text {nd }}$ edn., pp: 35-47 (http://whqlibdoc.who.int/publications/2010/9789241547925_eng.pdf?ua=1). 